\title{
Lighthouse: A Taxonomy-Based Solver Selection Tool
}

\author{
Kanika Sood \\ Computer and Information Science, \\ University of Oregon, Eugene, OR, \\ USA \\ kanikas@cs.uoregon.edu
}

\author{
Boyana Norris \\ Computer and Information Science, \\ University of Oregon, Eugene, OR, \\ USA \\ norris@cs.uoregon.edu
}

\author{
Elizabeth Jessup \\ Department of Computer Science, \\ University of Colorado, Boulder, \\ $\mathrm{CO}$, USA \\ jessup@cs.colorado.edu
}

\begin{abstract}
Linear algebra provides the building blocks for a wide variety of scientific and engineering simulation codes. Users face a world of continuously developing new algorithms and high-performance implementations of these fundamental calculations. In this paper, we describe new capabilities of our Lighthouse framework, whose goal is to match specific problems in the area of high-performance numerical computing with the best available solutions developed by experts. Lighthouse provides a searchable taxonomy of popular but difficult to use numerical software for dense and sparse linear algebra. Because multiple algorithms and implementations of the same mathematical operations are available, Lighthouse also classifies algorithms based on their performance. We introduce the design of Lighthouse and show some examples of the taxonomy interfaces and algorithm classification results for the preconditioned iterative linear solvers in the Parallel Extensible Toolkit for Scientific Computation (PETSc).
\end{abstract}

Categories and Subject Descriptors D.2.2 [Software Engineering]: Design Tools and Techniques

Keywords linear algebra, mathematical software, machine learning, taxonomy

\section{Introduction}

Solving large linear systems and computing eigenvalues are fundamental problems in high-performance scientific and engineering computing (HPC). In response to the need for high-performance algorithms, applied mathematics and computer science researchers have created a number of comprehensive numerical software packages that are widely used

Permission to make digital or hard copies of all or part of this work for personal or classroom use is granted without fee provided that copies are not made or distributed for profit or commercial advantage and that copies bear this notice and the full citation on the first page. Copyrights for components of this work owned by others than ACM must be honored. Abstracting with credit is permitted. To copy otherwise, or republish, to post on servers or to redistribute to lists, requires prior specific permission and/or a fee. Request permissions from Permissions@acm.org.

SEPS'15, October 27, 2015, Pittsburgh, PA, USA

(c) 2015 ACM. 978-1-4503-3910-0/15/10 ..\$15.00

http://dx.doi.org/10.1145/2837476.2837485 today. Because of the number and complexity of the algorithms available in these packages, finding the most suitable solution to a particular problem is a nontrivial task, even for experts in numerical methods. For example, for the relatively limited problem of solving a dense system of linear equations, one numerical library LAPACK $[3,6]$ alone offers over 100 different routines.

Lighthouse is a framework for creating, maintaining, and using a taxonomy of available software for highly optimized matrix algebra computations. The taxonomy serves as a guide to HPC application developers seeking to learn what is available for their programming tasks, how to use it, and how the various parts fit together. In this paper, we briefly introduce the Lighthouse tool (described in more detail in [4]) then focus on new Lighthouse capabilities in the area of parallel iterative solvers for sparse linear systems in the Portable Extensible Toolkit for Scientific Computation (PETSc) [8-10]. PETSc has been used for modeling in many areas, ranging from acoustics [34] to brain surgery [7] to ocean dynamics [33].

\section{Design and Implementation}

The main components of the Lighthouse framework are illustrated in Figure 1. Lighthouse defines its software taxonomy using Django [1] models, which correspond to MySQL databases that store information about the different packages. Currently Lighthouse contains routines from LAPACK, SLEPc [5], and PETSc.

Lighthouse enables search to the user via three methods: guided search, advanced search and keyword search. In the guided search, users are asked detailed questions in order to describe the problem they wish to solve with Lighthouse. After answering all the questions, the user sees exactly one subroutine that corresponds to all the answers provided.Portions of the interface are generated automatically, based on the user's responses using Django dynamic forms and Django session framework. The keyword search interface supports keyword-based search of the taxonomy information. In it, Lighthouse supports auto completion of words and spelling correction. Finally, the advanced search is geared toward users who are familiar with the packages. Most of the dialogs 


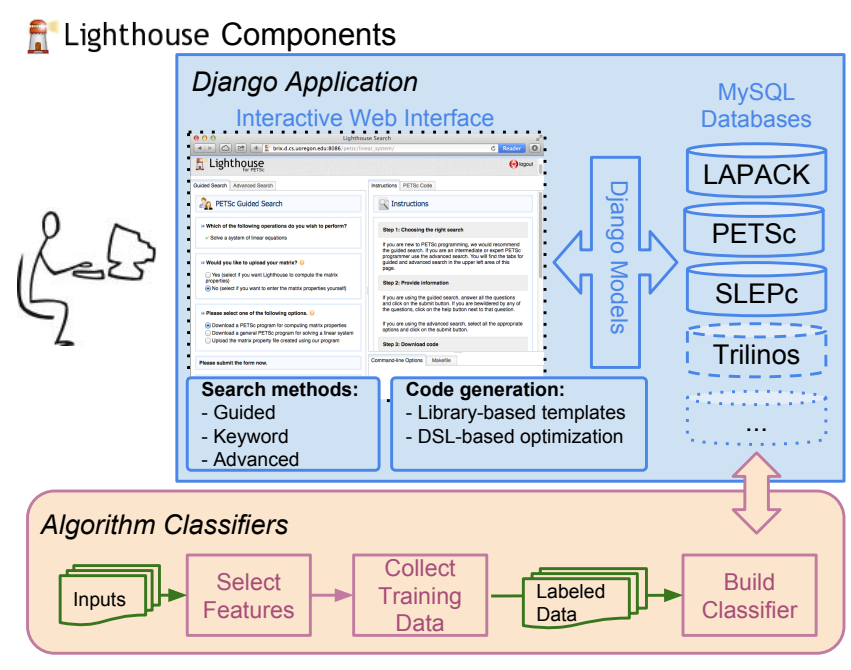

Figure 1. Lighthouse overview

are generated automatically based on data retrieved from the taxonomy database.

Lighthouse generates two types of code. First, given a specific search result, Lighthouse provides users with the option of generating a complete program that uses those function(s) correctly, including declarations and initialization of all data structures. Second, Lighthouse provides a separate experimental interface that allows users to define their own high-level linear algebra computations for dense and some sparse linear systems and vectors. Using a MATLAB-like language as input, Lighthouse interfaces with the BTO compiler $[11,32]$ to generate highly optimized $\mathrm{C}$ implementations that can be downloaded and used in larger applications.

\section{Taxonomy Search Interfaces}

We briefly illustrate the user interfaces of Lighthouse with an example for solving a large sparse linear system using PETSc. For each numerical library supported by Lighthouse, including PETSc, we have created decision trees with the questions used in the guided search interfaces. These trees are stored in databases, from which the actual interactive Web forms are generated dynamically. The details of the taxonomy creation and representation as well as the existing support for LAPACK are described in more detail in [29]. Significant portions of the user interface are also automatically generated from databases containing the decision tree questions and answers. For LAPACK, for example, each question in the guided search is determined dynamically upon processing the user's answer to the previous question. For PETSc, the guided search is somewhat simpler because there are fewer distinctions to be made among methods based on data structure properties (i.e., all solvers work with many matrix formats of any precision).

Figure 2 shows the guided search dialog in the Lighthouse PETSc interface, which has a series of questions for the user. It is an interactive system that enables users to generate as well as download PETSc programs for solving sparse linear systems. To begin, the user has the option to upload a coefficient matrix and have Lighthouse compute its properties or features. If the user chooses this option, Lighthouse uses the matrix properties in a machine learning classification process (described in Section 4) to predict a well-performing solver for the given system. If a user does not wish to upload a matrix, Lighthouse also provides a PETSc program for computing matrix properties or a generic PETSc program for solving a linear system.

The code generated by Lighthouse as a result of the guided search is shown in Figure 3. Lighthouse currently generates code templates in $\mathrm{C}$ or Fortran 90 for the functions returned in the search. The template is a complete program, including all variable declarations and correct invocation of library functions, many of which take very large numbers of parameters. The template program is easy to modify as it is divided into multiple subprograms.

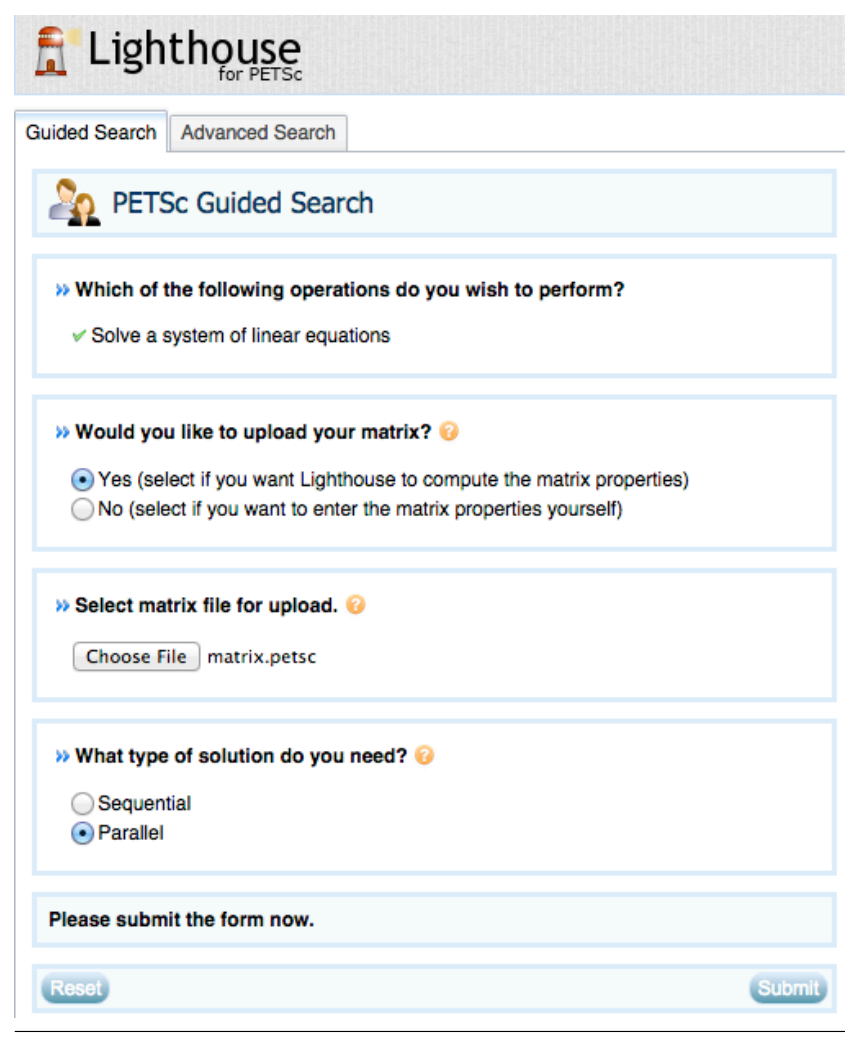

Figure 2. PETSc guided search interface

\section{Sparse Linear Solver Classification}

Large sparse linear system solution is a fundamental computational step in many scientific and engineering applications. Users generally choose one of many iterative methods for approximating the solution to a system (subsequently, we refer to these methods as solvers). Individual solver convergence and performance is highly dependent on the specific input problem characteristics and can be difficult to predict. 


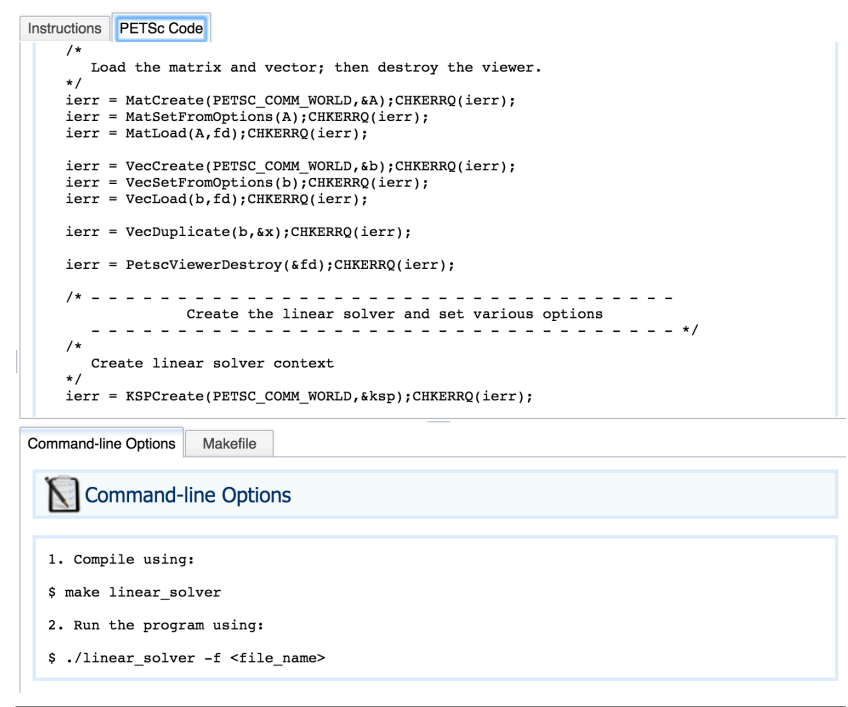

Figure 3. PETSc code template resulting from guided search

Lighthouse automates the performance-based solver selection process by employing machine learning (ML) techniques to classify solvers based on a training set generated with over 1,000 linear systems arising from different applications. We applied several supervised ML techniques to help make this decision based on relatively few, easily computable properties of the input system. Supervised learning involves determining a classification based on a set of already classified data. These data are split into training and testing sets. We performed a $66 \%-34 \%$ split for the training and testing sets, respectively. The training set is used to build the classifier and the testing set is used to verify the accuracy of the classifier. For our experiments, this process was repeated 10 times (10-fold cross validation), each time with a different subset as the testing set. Our ultimate goal is to provide a scalable and automated approach for choosing an efficient and accurate solver for a given linear system by classifying linear solvers using several ML methods. In the past, there have been several attempts made to use machine learning to identify "good" solvers in the context of parallel nonlinear PDE solution $[12,13,28]$. In the area of sparse linear system solution, several machine learning approaches are used to classify a limited set of methods [14, 19, 22, 35]. The success of these more limited multi-solver methods motivates the addition of solver classification to Lighthouse, which we describe next. The novelty of our work is that it introduces a robust, generalizable approach to solver classification, which can be fully automated and hence can track the evolution of the software packages it supports.

Linear solver classification in Lighthouse is performed as a sequence of steps. Note that this process is generalizable to other problems beyond sparse linear system solution. The features used in building the solver classifiers include matrix properties such as simple (norm-like) quantities, vari- ance (heuristics estimating how different matrix elements are), normality (estimates of the departure from normality), structure (nonzero structure properties), and spectral properties. Because computing so many matrix properties (in our case 68) while an application is executing is too costly, we also performed feature set reduction, removing the features that do not contribute significantly to the process of deciding the best solver for a given linear system. Feature set reduction brings down the overall cost of the process for building and using the classifiers. The next step involves building classifiers by using several machine learning methods in Weka [21]: BayesNet [15], k-nearest neighbor [18], Alternate Decision Trees [20], Random Forests [16], J48 [30], Support Vector Machines (SVM) [17], Decision Stump [24] and LADtree [23] methods. We compared the performance of these methods to select the one that most efficiently produces the best (most accurate) solver classification.

\subsection{Classification Results}

To generate the input dataset used by the machine learning methods to classify solvers, we measured the performance of several solver and preconditioner combinations in PETSc version 3.5.3 on a Blue Gene/Q supercomputer on more than 1,000 linear systems from the University of Florida matrix collection [2]. Because the University of Florida matrices are not very large, each experiment was run sequentially on a single node. For each input, we computed the linear system's features, then solved the system using a specific solver configuration and measured the execution time, resulting in 4,648 data points (solver execution times).

Next, we used a subset of the data as a training set to build classifiers using machine learning methods. We selected the one that most accurately and efficiently classifies solver performance. The accuracy of classifiers was verified using the remaining data as the testing data set. The performance of the best classifier for predicting good solvers was verified using 10-fold cross validation with a $66 \%-34 \%$ data split for training and testing sets respectively. In Lighthouse, we focus only on true positives (i.e., best-performing solvers predicted as best-performing). Among all machine learning methods we used, for the 66\%-34\% data split, BayesNet performed the best, with accuracies of $89.2 \%$ with the full feature set. With Reduced Set 1 and Reduced Set 2, BayesNet again outperformed the other classifiers achieving an accuracy of $86.9 \%$ and $86.6 \%$ accuracy respectively. With 10 fold cross validation, BayesNet produced the best accuracy of $87.6 \%$ with 68 input features. Using only eight computationally inexpensive features, the BayesNet-based classifier predicted good (well-performing) solvers correctly $86.9 \%$ of the time and using reduced set 2 features, BayesNet achieved an accuracy of $86.4 \%$.

Figure 4 is a radar chart that shows the true positive prediction accuracy of the six machine learning methods we tested for the full and reduced feature sets. The radar chart contains ten radial axes, with values ranging between $0 \%$ 
and $100 \%$ from the center to the perimeter in increments of $10 \%$. An accuracy of $100 \%$ indicates a classifier that predicts the good classifiers correctly each time. An accuracy of $0 \%$ indicates a classifier that fails to predict good classifiers in each case.

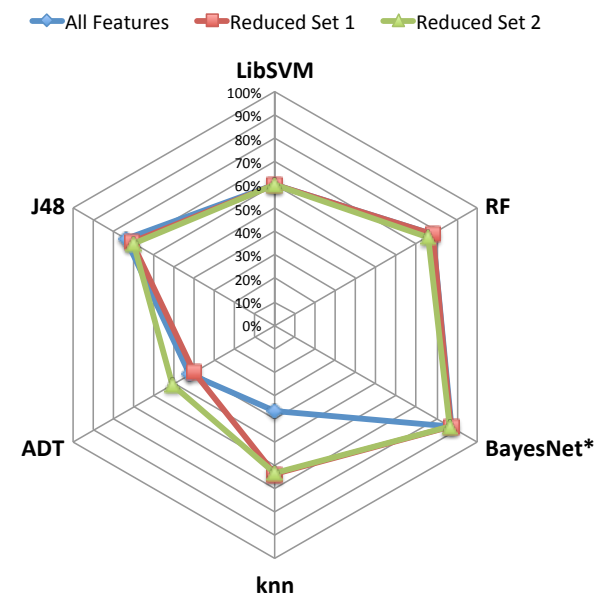

Figure 4. Machine learning method accuracy comparison.

In addition to the accuracy of the classifier, we also considered the time it took to build the classifier, which can vary by orders of magnitude between different methods. The BayesNet method, which has the highest accuracy, took a fraction of a second to build the PETSc solver classifier on an Intel Core i5 MacBook Pro. The slowest method was LibSVM, taking over a minute to build the classifier. For our experiments we considered problems from various domains such as electro-magnetics, acoustics, computational fluid dynamics, circuit simulation, power network and others. Therefore, the conclusions obtained from our results are not specific to any one domain and are applicable to various domains widely available.

\subsection{Using the Classification Results to Select Solvers}

When a user is able to provide an example linear system from a specific application, Lighthouse can compute the reduced feature set and use the previously generated classifier to return a list of solver configurations that are likely to perform well. In future work, we also plan to enable users to check whether a user-specified solver configuration is likely to perform well.

\section{Evaluation}

As Lighthouse continues to grow, we plan to apply formal methods of user testing from human computer interaction (e.g., [25-27, 31]) to evaluate its usability. For example, we will employ techniques for interface developers, such as cognitive walkthrough [31] and heuristic evaluation [26, 27], and we will continue to use those directed at users, such as thinking aloud [25].

To date, we have carried out thinking aloud evaluations of Lighthouse in graduate courses at the University of Colorado
Boulder. The clients had education and expertise in fields of engineering (aerospace, electrical, civil), mathematics, computer science, and computational science and varying expertise in linear algebra and programming. These clients discovered a number of usability issues and also made very good suggestions for improvement of Lighthouse. These preliminary evaluations demonstrate the value of user feedback.

\section{Conclusions and Future Work}

At present, Lighthouse for PETSc has an efficient navigation system that allows users to generate, download and extend PETSc programs for solving large sparse linear systems. Because there are thousands of valid linear solver configurations, we have added to Lighthouse a machine learningbased solver classification. It can substantially improve developer productivity because there is no need to experiment with various solvers in an ad hoc fashion, and it can improve application performance.

Future work includes extending the present Lighthouse interface to increase automation of the solver selection process, which will enable the regular regeneration of the classifiers as more data are collected from different applications, both user-provided and our own. Our current analysis considers only sequential runs; we will apply the same approach for parallel cases, and we will further expand the number of supported solver configurations.

\section{Acknowledgments}

This work is supported by the U.S. Department of Energy Office of Science (Contract No. DE-SC0013869) and by the National Science Foundation (NSF) awards CCF-1219089, CCF-155063 and CCF-1550202.

\section{References}

[1] Django. https://www.djangoproject.com/, 2015.

[2] The University of Florida Sparse Matrix Collection. http: //www.cise.ufl.edu/research/sparse/matrices/, 2015.

[3] LAPACK - Linear Algebra PACKage. http://www.netlib.org/lapack/, 2015.

[4] The Lighthouse Project. http://lighthousehpc.github.io/lighthouse/, 2015.

[5] Scalable Library for Eigenvalue Problem Computations (SLEPc). http://www.grycap.upv.es/slepc/, 2015.

[6] E. Anderson, Z. Bai, J. Dongarra, A. Greenbaum, A. McKenney, J. Du Croz, S. Hammerling, J. Demmel, C. Bischof, and D. Sorensen. LAPACK: A Portable Linear Algebra Library for High-performance Computers. In Proceedings of the 1990 ACM/IEEE conference on Supercomputing, pages 2-11. IEEE Computer Society Press, 1990.

[7] Y. Ataseven, Z. Akalın-Acar, C. Acar, and N. G. Gençer. Parallel implementation of the accelerated BEM approach for 
EMSI of the human brain. Medical \& Biological Engineering \& Computing, 46(7):671-679, 2008.

[8] S. Balay, W. D. Gropp, L. C. McInnes, and B. F. Smith. Efficient management of parallelism in object oriented numerical software libraries. In E. Arge, A. M. Bruaset, and H. P. Langtangen, editors, Modern Software Tools in Scientific Computing, pages 163-202. Birkhäuser Press, 1997.

[9] S. Balay, S. Abhyankar, M. F. Adams, J. Brown, P. Brune, K. Buschelman, L. Dalcin, V. Eijkhout, W. D. Gropp, D. Kaushik, M. G. Knepley, L. C. McInnes, K. Rupp, B. F. Smith, S. Zampini, and H. Zhang. PETSc users manual. Technical Report ANL-95/11 - Revision 3.6, Argonne National Laboratory, 2015. URL http://www.mcs.anl.gov/petsc.

[10] S. Balay, S. Abhyankar, M. F. Adams, J. Brown, P. Brune, K. Buschelman, L. Dalcin, V. Eijkhout, W. D. Gropp, D. Kaushik, M. G. Knepley, L. C. McInnes, K. Rupp, B. F. Smith, S. Zampini, and H. Zhang. PETSc Web page, 2015. URL http://www.mcs . anl.gov/petsc.

[11] G. Belter, E. R. Jessup, I. Karlin, and J. G. Siek. Automating the generation of composed linear algebra kernels. In SC '09: Proceedings of the Conference on High Performance Computing Networking, Storage and Analysis, pages 1-12, New York, 2009. ACM. ISBN 978-1-60558-744-8. .

[12] S. Bhowmick, P. Raghavan, L. McInnes, and B. Norris. Faster PDE-based simulations using robust composite linear solvers. Future Generation Computer Systems, 20(3): 373-387, 2004.

[13] S. Bhowmick, D. Kaushik, L. McInnes, B. Norris, and P. Raghavan. Parallel adaptive solvers in compressible PETSc-FUN3D simulations. In Proceedings of the 17th International Conference on Parallel CFD, 2005.

[14] S. Bhowmick, V. Eijkhout, Y. Freund, E. Fuentes, and D. Keyes. Application of alternating decision trees in selecting sparse linear solvers. In Software Automatic Tuning, pages 153-173. Springer, 2010.

[15] C. Bielza and P. Larrañaga. Discrete Bayesian network classifiers: A survey. ACM Comput. Surv., 47(1):5:1-5:43, July 2014. ISSN 0360-0300. . URL http://doi.acm.org/10.1145/2576868.

[16] L. Breiman. Random Forests. Mach. Learn., 45(1):5-32, Oct. 2001. ISSN 0885-6125. . URL http://dx.doi .org/10.1023/A: 1010933404324.

[17] C. Cortes and V. Vapnik. Support-vector networks. Machine learning, 20(3):273-297, 1995.

[18] P. Cunningham and S. J. Delany. k-nearest neighbour classifiers. Multiple Classifier Systems, pages 1-17, 2007.

[19] P. R. Eller, J.-R. C. Cheng, and R. S. Maier. Dynamic linear solver selection for transient simulations using machine learning on distributed systems. In Proceedings of the IEEE Parallel and Distributed Processing Symposium Workshops (IPDPSW), pages 1915-1924. IEEE, 2012.

[20] Y. Freund and L. Mason. The Alternating Decision Tree Learning Algorithm. In Proceedings of the Sixteenth International Conference on Machine Learning, ICML '99, pages 124-133, San Francisco, CA, USA, 1999. Morgan Kaufmann Publishers Inc. ISBN 1-55860-612-2. URL http: //dl.acm.org/citation. cfm?id=645528.657623.

[21] M. Hall, E. Frank, G. Holmes, B. Pfahringer, P. Reutemann, and I. H. Witten. The WEKA Data Mining Software: An Update. SIGKDD Explorations, 11, 2009.

[22] A. Holloway and T.-Y. Chen. Neural networks for predicting the behavior of preconditioned iterative solvers. In Computational Science-ICCS 2007, pages 302-309. Springer, 2007.

[23] G. Holmes, B. Pfahringer, R. Kirkby, E. Frank, and M. Hall. Multiclass alternating decision trees. In Machine learning: ECML 2002, pages 161-172. Springer, 2002.

[24] W. Iba and P. Langley. Induction of one-level decision trees. In Proceedings of the Ninth International Conference on Machine Learning, pages 233-240, 1992.

[25] C. Lewis. Using the thinking-aloud method in cognitive interface design. Technical Report IBM Research Report RC 9265, IBM, Yorktown Heights, NY, 1982.

[26] J. Nielsen. How to conduct a heuristic evaluation. http://www. useit.com/papers/heuristic/ heuristic_evaluation.html, 2007.

[27] J. Nielsen. Ten usability heuristics. http://www.useit. com/papers/heuristic/heuristic_list.html, 2007.

[28] B. Norris, L. C. McInnes, S. Bhowmick, and L. Li. Adaptive numerical components for PDE-based simulations. PAMM, 7 (1):1140509-1140510, 2007.

[29] B. Norris, S.-L. Bernstein, R. Nair, and E. Jessup. Lighthouse: A user-centered Web system for linear algebra software. Elsevier Journal of Systems and Software (JSS): Special Issue on Software Engineering for Parallel Systems, 2015. To appear; arXiv preprint arXiv:1408.1363.

[30] R. Quinlan. C4.5: Programs for Machine Learning. Morgan Kaufmann Publishers, San Mateo, CA, 1993.

[31] J. Rieman, M. Franzke, and D. Redmiles. Usability evaluation with the cognitive walkthrough. http://www.acm.org/sigs/sigchi/chi95/ Electronic/documnts/tutors/jr_bdy.htm, 2004.

[32] J. G. Siek, I. Karlin, and E. R. Jessup. Build to order linear algebra kernels. In Workshop on Performance Optimization for High-Level Languages and Libraries (PO HLL 2008), pages 1-8, April 2008.

[33] A. D. T. van Scheltinga, P. G. Myers, and J. D. Pietrzak. A finite element sea ice model of the Canadian Arctic Archipelago. Ocean dynamics, 60(6):1539-1558, 2010.

[34] J. Wang, Y. Wang, W.-k. Hu, and J.-k. Du. The high performance of finite element analysis and applications of surface acoustic waves in finite elastic solids. In Piezoelectricity, Acoustic Waves, and Device Applications, 2008. SPAWDA 2008. Symposium on, pages 66-71. IEEE, 2008.

[35] S. Xu and J. Zhang. SVM Classification for Predicting Sparse Matrix Solvability with Parameterized Matrix Preconditioners. Technical Report 458-06, University of Kentucky, 2006. 